\title{
Deciding on prosthodontic treatment in children with inherited dental abnormalities: should psychological or dental well-being take precedence?
}

SADJ September 2018, Vol. 73 No. 8 p532 - p535

LM Sykes ${ }^{1}$, R Doolabh ${ }^{2}$

"It is only with the heart that one can see rightly. What is essential is invisible to the eye"

(The Little Prince, by Antoine De Saint-Exupery).

\section{INTRODUCTION}

Children with inherited dental anomalies have many complex problems that may interfere with their physical, mental and psychological well-being. ${ }^{1,2}$ The anomalies generally present early in life as isolated conditions, or associated with other defects, and may require immediate as well as prolonged multidisciplinary management. ${ }^{2}$

The spectrum of defects is diverse, as is the range of deformities that can manifest in the head and neck region. ${ }^{1}$ Common to all is their potential to have "profound negative consequences for the individual and their families, ranging from aesthetic concerns that impact on their self-esteem, to masticatory difficulties, tooth sensitivity, financial burdens, and protracted dental treatment." 2

\section{PSYCHOLOGICAL IMPACT OF DENTO-FACIAL DEFECTS}

Over the years, the concept of beauty has changed vastly and differs between cultures, yet it remains an elusive notion that is "easily recognised, often sought after, revered and sometimes seen as a personal virtue". ${ }^{3}$

Currently, the media has a large influence on the perception of beauty by defining it in terms of specific physical attributes such as a healthy toned body, facial symmetry, a good complexion, a bright smile with well aligned white teeth, large eyes, a youthful complexion, and an image of vitality.

Author affiliations:

1. Leanne M. Sykes, MDent (Pros), Dip Res Ethics (Irensa),

Dip ForOdont, Department of Prosthodontics, University of Pretoria.

2. Rajesh Doolabh, BDS, MSc, PDBA, MDent(Pros), Department of Prosthodontics, University of Pretoria.

Corresponding author: L.M. Sykes

Department of Prosthodontics, University of Pretoria.

Email: leanne.sykes@up.ac.za
There is a great emphasis on the face as the most valued aspect of human beauty, as it is the one feature which cannot be concealed, and is often used by others to describe a person.

People instinctively and preferentially look at the faces in photographs, and studies have even found that viewing attractive faces activates brain structures associated with the reward centres in the brain such as those related to food and money. These perceptions are so embedded in society that facial attractiveness has even been defined as "a social necessity" or "a gift from God". ${ }^{4}$

Beauty also has positive re-inforcement values such that those who possess this "gift" are also perceived to have other positive personality traits and seem to be liked more, and to benefit from preferential treatment in all aspects of life, and throughout their lives. ${ }^{5}$ In some spheres, appearance is even used as a social marker determining status and acceptance. ${ }^{3}$ This in turn has led to a distorted level of importance being placed on attaining the "ideal".

Many believe that beauty is the bearer of identity, character and intelligence, ${ }^{6}$ and that being beautiful affords both men and women advantages in school, and in their chosen careers. Attractive employees often receive more promotions and salary raises than their less attractive counterparts, good-looking students are more popular, are considered to be more intelligent and often do better in school. ${ }^{4,7}$

Some have gone so far as to state that "there is a universal standard for facial beauty based on ideal proportions that are directly related to the divine proportion. Organisms that conform to these proportions are not only beautiful, but also biologically healthy". ${ }^{6}$ Others believe that facial symmetry is an important determinant of good looks, and that body symmetry is directly related to mental ability, despite the fact that mild asymmetry is normal. ${ }^{4,8}$

These unrealistic notions and pursuit of perfection have become an obsession and a multi-million rand industry with many individuals resorting to extreme measures in the attempt to attain the perfect look. ${ }^{7}$ 
On the other hand, reverse stereotypes about less attractive individuals also exist. Those with abnormal facial features are often discriminated against, and considered to be dishonest, less capable, less intelligent, aggressive and less successful based on unsubstantiated assumptions. ${ }^{9}$

At the same time, a person's own perception of their facial appearance is of great importance, and thus it is not surprising that patients with craniofacial defects may experience difficulties adapting to their deformity, no matter how mild or severe. This erroneous appraisal can interfere with their personal lives, having a negative psychosocial impact, with increased self-consciousness, low self-esteem, behavioural disorders, poor social interactions, and emotional distress. ${ }^{1,4}$

\section{PARENTAL PRESSURE TO TREAT}

Considering that the "face is often the foundation of judgement when assessing appearance", ${ }^{3}$ any facial deformity may be considered a "social disability" that impacts on the individual, as well as their families, who observe how it is noticed and reacted to by strangers. ${ }^{4}$

Children with a different dentofacial appearance are often subject to unsympathetic teasing, harassment, and malicious verbal and physical intimidation. ${ }^{4}$ The impact of this may be seen by the child developing habits and behaviour to try to conceal the mouth, such as covering with their hands, tensing their oral musculature, avoiding smiling, avoiding eye contact, limiting speaking and communicating with others, social embarrassment and a negative body image. ${ }^{10}$

It is thus not surprising that parents who wish to spare their youngsters from this psychological distress will seek and demand any form of medical, dental or cosmetic intervention at an early age, often just before the child is about to begin school.

\section{PROSTHODONTIC MANAGEMENT}

The face, especially the oral region is central to all contact and interpersonal communication. The mouth itself is instrumental in chewing, speaking and expressing emotions and thoughts, all activities necessary in daily life.

Facial disfigurement may be congenital, traumatic or infectious. The commonly seen congenital defects include cleft lip and/or palate, partial anodontia, amelogenesis imperfecta, dentinogensis imperfecta, and ectodermal dysplasia.

These problems may present as dental deviations in size, position, appearance and number of teeth, as well as facial variances such as decreased vertical dimension, decreased facial support, lack of occlusion, incompetent lips, altered speech, tooth wear and sensitivity. ${ }^{11}$

Many clinicians (and parents) feel that the dental concerns should be addressed as soon as they present in order to spare the patients (and their families) from negative psychological consequences. ${ }^{2}$ However, no interventions should be contemplated before there has been a multi-disciplinary consultation, and a comprehensive treatment plan has been drafted outlining the immediate, short term and anticipated future needs. In some situations immediate treatment soon after birth is required to aid feeding and/ or breathing.

Any other early intervention, especially for aesthetic concerns alone, needs careful consideration. While it may be beneficial in reassuring the parents that they have help and support, it must not be to the detriment of future treatment or oral health.

The more difficult prosthodontic decisions concern those children who are about to begin school. Parents and teachers often put pressure on dentists to carry out treatment that will improve the child's appearance and spare them from teasing and stigmatization.

Restorative options may be very limited and will depend on the child's age, their particular anomaly, the appearance, quality, distribution and number of teeth present, and the radiographic assessment of all developing, but as yet unerupted permanent teeth. Conceding to unrealistic expectations and demands to treat can carry physical and psychological risks for the patients. ${ }^{3}$

Nowak cautioned that "Any clinicians who decide to treat paediatric patients with congenital defects must be knowledgeable in growth and development, behaviour management, techniques for fabrication of specialised prostheses, conservative modification of existing teeth, have the ability to motivate the patient and the parent in the use of the prosthesis, be responsible for the follow-up maintenance, modification and replacement of the prosthesis and have a plan for the long-term definitive treatment". ${ }^{12}$

If not competent in all of these areas, the practitioner should consult with other specialists, or refer the patient if necessary. Regardless of the individual clinician's experience and expertise, management of all children with congenital defects is best handled by a multidisciplinary team.

This may comprise a paediatric dentist, prosthodontist, orthodontist, maxillofacial surgeon, ear nose and throat specialist, speech therapist, psychologist, social worker, and should include the parents, with each performing care in their particular field if and when it is indicated.

There are no guidelines as to the best time to begin treatment. Some clinicians may feel that an initial appliance should be placed before the child begins school to give them a reasonably normal appearance and allow them time to adapt. ${ }^{12}$ However, this decision must be based on their ability to manage the child, the oral condition at that time as well as the child's ability to withstand dental procedures - including consideration of the need for general anaesthesia.

The parents may also facilitate or impede the success of the treatment. It is important that they, as well as the patient, understand what is involved in each procedure, the anticipated outcome, what the appliance will look like, how it will benefit the patient, how much adaptation may be needed, how to care for it, the number and frequency 
of visits that will be needed for post insertion modifications or replacement, possible risks or damage that the treatment may cause to any teeth or other oral structures, and limitations of the treatment. ${ }^{12}$

A good outline of possible prosthodontic options for children with congenital dental defects was presented by Kanaparthy and Kanaparthy (2015). ${ }^{13}$ These include:

\section{Fixed partial dentures}

These are rarely used because these children often have a limited number of teeth, and their position and spacing is seldom ideal. There is risk of pulp exposure when cutting young teeth with large pulp chambers. Rigid fixed prostheses may interfere with jaw growth, especially if they cross the midline.

\section{Individual crowns}

While individual crowns will not restrict jaw growth they still carry the risk of pulpal exposure in young teeth. Teeth may also not be fully erupted and thus have short clinical crowns which could compromise retention.

In the anterior region, the crown margins may become exposed once the teeth have fully developed and the unaesthetic crowns will then need to be replaced.

Pupal exposure in patients with dentinogenesis imperfecta is a problem as endodontic treatment is extremely complicated due to pulpal obliteration. ${ }^{2}$

\section{Direct composite restorations and veneers}

These are ideal for restoring normal form, function and aesthetics to malformed teeth. They are minimally invasive and easily replaced when needed.

\section{Partial and complete removable prostheses}

These are the most common form of treatment as many congenital defects present with anodontia, hypodontia, malformed or malpositioned teeth.

Removable appliances include partial dentures, complete dentures, removable appliances carrying an obturator or speech bulb extension as well as removable appliance used in conjunction with orthodontic mechanisms.

Child patients requiring complete dentures often have underdeveloped ridges and will struggle with retention, speech and mastication when wearing a complete removable denture.

\section{Overdentures}

If there are any teeth, no matter how small, misshapen or deformed, they will help retain and stabilize an overdenture. The added advantage of overdentures especially in patients with cleft palate defects is that retention of teeth helps maintain and preserve the alveolar bone, while the denture itself can be used to bulk out an underdeveloped maxilla.
Overdentures are also ideal in patients with dentine dysplasia, as the teeth usually have shortened roots and are thus lost at an early stage. The overdentures are then easily converted to complete dentures. ${ }^{2}$ They are similarly suited to children suffering from anodontia.

\section{Bleaching and micro-abrasion}

These have been used in patients with severe tooth discolouration prior to placing composite resin or bonded ceramic veneers or full coverage restorations.

Some patients with Amelogensis imperfecta may require periodontal surgery to extend crown lengths or reduce hyperplastic tissue, ${ }^{2}$ but this is particularly invasive and traumatic for a child.

\section{Implant supported prostheses}

There is much literature to support the psychological and physical benefits of implant supported prostheses in patients with congenital defects.

However the consensus opinion is that they should only be placed after maximum jaw growth has occurred which is estimated to be at around 15 years of age. ${ }^{13}$

There are many prosthodontic options available to treat children with inherited dental anomalies. While the possible improved aesthetics and assumed psychological benefits of intervening at an early age may tempt clinicians to treat, there is a need to be aware that there are also many less obvious dental complications as well as physical and psycho-social disadvantages. These include, but are not strictly limited to the following:

- unretentive prostheses that may hamper mastication or speech and be socially embarrassing to the child. Trying to force them to wear such an appliance for aesthetic purposes could traumatize them more than the psychological issues already associated with having a deformity.

- they invariably miss a fair amount of school time, extra-curricular activities and social functions while they are undergoing treatment and recovery. This can add to their already compromised academic and social development, and could further impact on their low self-esteem.

- they may experience extreme discomfort, and suffer from iatrogenic dental damage as a result of the tooth preparations required to accommodate a prosthesis.

- appliances need to be regularly adjusted to permit continued tooth development and eruption. If this is not monitored closely they can cause extensive damage and even tooth loss which will jeopardise future treatment options. Who is responsible to ensure the child returns for these scheduled visits, the clinician who inserts the prosthesis or the parents, and who should be held liable for damage caused by lack of compliance?

- surgical procedures carry a risk of scarring and tissue contraction, which result in a poor denture foundation when compared with normal healthy oral mucosa.

- root resorption from accelerated orthodontic tooth movement, especially if it is carried out without consulting specialists in the field, or from manipulation of teeth with deficient root structures. 
- instilling a fear of dentists into a child at such an early age may impact on how they view their oral condition and therapy for the rest of their lives.

- finally, there is the ethical issue of whether it is justified to expose children to multiple radiographic procedures, frightening and often painful dental treatment, or repeated sessions of sedation and general anaesthesia.

\section{CONCLUSIONS}

In an ideal world dentists would be able to provide prosthodontic treatment that would improve aesthetics and function, not compromise oral health and simultaneously address the child's psycho-social needs.

However, ideal seldom exists. In those situations where there is a dilemma in deciding between prosthodontic intervention and delayed treatment, it may be necessary to also consider the patient's "quality of life". This includes physical health, personal circumstances, social relationships and functionality.

The authors cannot dictate which treatment approach should take precedence. Is it better to succumb to parental pressure and treat the dento-facial condition at any cost in order to improve the appearance and psychological well-being of the child, or should there be minimal initial intervention, if doing so will benefit long term future oral and dental health?

The literature has as many proponents as opponents for each argument - with some expressing extreme views such as:

- "We as dentists can harmonize and normalize the face and health of those with unattractive facial features. In so doing we are able to offer these children a life they may not otherwise achieve: an equal opportunity for health, happiness and success. We wield the power within our fingertips to intervene professionally and change their lives in a positive way. This is a supreme gift bestowed upon us - a gift that we should use wisely and responsibly." 6

As opposed to:

- Until the world is able to see that true beauty cannot and should not be defined or outlined by a perfect set of measurements or in a finite list of attributes, it will leave many people the poorer as they will miss out on appreciating the talents, virtue, and soul of their fellow human beings.

Beauty does not reside in the eyes but rather in the heart. It manifests itself in the acts of kindness and compassion shown to others, in how people relate, learn from and teach each other, and in the respect they show for all of creation. In that respect, everyone is already beautiful. ${ }^{7}$

We will welcome feedback and opinions from colleagues. We guarantee that confidentiality and anonymity will be maintained and look forward to present these comments in the form of a lively debate, in a follow up publication.

\section{References}

1. Singh V, Moss TP. Psychological impact of visible differences in patients with congenital craniofacial anomalies. Progress in Orthodontics 2015;16(5):DOI 10, 1186/s40510-015-0078-9.

2. American Academy of Pediatric Dentistry. Guideline on dental management of heritable dental developmental anomalies. Accessed at: www.aapd.org/media/policies_guidelines/G_ OHCHeritable1.pdf: Council of Clinical Affairs, American Academy of Pediatric Dentistry 2013. p. 16-7; Accessed on 12-12-2017.

3. Ford P. Beauty in different cultures. 2009. Accessed at: http:// paulford.com/beauty-in-different-cultures/

Accessed on: 15-08-2012.

4. Naini F, Gill DS. Facial aesthetics: 1. Concepts and canons. Dental Update 2008:102-7.

5. Sigall H. Ostrove N. Beautiful but dangerous: Effects of offender attractiveness and nature of the crime on juridic judgement. $J$ Personality and Social Psychology. 1975;31(3):410-4.

6. Jefferson Y. Facial beauty - Establishing a universal standard. IJO. 2004;15(1):9-22.

7. Kuchinsky C. What is true beauty? - The Beauty Biz - Article Accessed at: www.thebeautybiz.com/163/article/body/truebeauty2009; Accessed on: 18-01-2018.

8. Banks GC, Batchelor JH, McDaniel MA. Smarter people are (a bit) more symmetrical: A meta-analysis of the relationship between intelligence and fluctuating asymmetry. Intelligence 2010;38:393-401.

9. Bull R. David I. Appearance and aesthetics in oral health. Community Dentistry Oral Epidemiology 1980;8:244-56.

10. Davis LG. Ashworth PD, Spriggs LS. Psychological effects of aesthetic dental treatment. J Dentistry. 1998;17:99-108.

11. Hickey AJ. Salter M. Prosthodontic and psychological factors in treating patients with congenital and craniofacial defects. JPD. 2006;95(5):392-6.

12. Nowak AJ. Dental treatment for patients with Ectodermal Dysplasia. Birth Defects 1988;24(24):3-52.

13. Kanaparthy A, Kanaparthy R. A First look: Determinants of dental care for Ectodermal Dysplasia patients. IOSR J of Dental and Medical Sciences. 2015;14(10):69-72. 